\title{
PROPERTIES OF EPITAXIAL ZNO THIN FILMS FOR GAN AND RELATED APPLICATIONS
}

\author{
H. Shen*, M. Wraback*, J. Pamulapati*, S. Liang**, C. Gorla**, and Y. Lu** \\ *U.S. Army Research Laboratory, Sensors and Electron Devices Directorate, \\ AMSRL-SE-EM, 2800 Powder Mill Rd., Adelphi, MD 20783-1197 \\ pshen@arl.mil \\ **Dept. of Electrical and Computer Engineering, Rutgers University, \\ Piscataway, NJ 08855-0909
}

\begin{abstract}
In this paper, we present a detailed study of high quality (110) $\mathrm{ZnO}$ films, epitaxially grown on R-plane sapphire substrates by metal-organic chemical vapor deposition (MOCVD). The

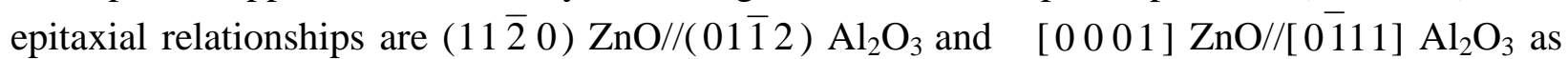
confirmed by X-ray diffraction $(\theta-2 \theta$, and $\phi$-scan) and high-resolution cross-sectional transmission electron microscopy (HR-TEM). Low temperature photoluminescence (PL) indicates the $\mathrm{ZnO}$ thin films are almost strain free. Optical absorption and reflection measurements with linearly polarized light indicate a strong optical anisotropy. The polarization rotation towards the $\mathrm{C}$-axis associated with the optical anisotropy is utilized to demonstrate an optically addressed ultra-fast, ultraviolet light modulator.
\end{abstract}

\section{INTRODUCTION}

High quality zinc oxide ( $\mathrm{ZnO})$ films are useful for many applications. One of the more recent applications is the use of $\mathrm{ZnO}$ as a substrate for growth of $\mathrm{GaN}$ based materials[1-3] since the lattice mismatch between $\mathrm{GaN}$ and $\mathrm{ZnO}$ is relatively small. Due to a lack of low cost $\mathrm{ZnO}$ substrates, high-quality $\mathrm{ZnO}$ buffer layers on other substrates are of particular interest. In addition, it is also possible to develop UV lasers and modulators from these $\mathrm{ZnO}$ films.[4,5]

The most common technologies used for depositing $\mathrm{ZnO}$ films are laser ablation and sputtering.[6] Improvements have been made through triode sputtering and other new deposition techniques. Despite these advancements, there are still some problems associated with sputtering. Metal-organic chemical vapor deposition (MOCVD)[7,8] is an alternative technique for growth that has advantages such as chemical and thermodynamic dependent growth, control at the atomic level, large area deposition, and the possibility of different in situ doping processes. While most research has focused on $\mathrm{ZnO}$ films grown on (0001) oriented sapphire substrates (i.e. C-plane $\mathrm{Al}_{2} \mathrm{O}_{3}$ ), less research has been done on $\mathrm{ZnO}$ grown on other planes. In this paper we report a detailed study of the properties of $\mathrm{ZnO}$ grown on (0112) oriented sapphire (R-plane). 


\section{SAMPLE GROWTH}

The samples used in this study were grown by MOCVD. R-plane sapphire was used as the substrate. The precursors used were DiEthyl Zinc (DEZn - $\left.\left(\mathrm{C}_{2} \mathrm{H}_{5}\right)_{2} \mathrm{Zn}\right)$ and $\mathrm{O}_{2}$. Typical growth conditions are as follows: chamber pressure of 20 to 50 torr; a growth temperature of 250 to $600{ }^{\circ} \mathrm{C}$; and a total carrier gas flow from the top of 5000 to $15000 \mathrm{sccm}$. The thickness of the sample is $1 \mu \mathrm{m}[9]$.

\section{STRUCTURAL PROPERTIES}

Figure 1 shows the $\mathrm{x}$-ray $\theta-2 \theta$ scans from the sample. From the scan we found that $\mathrm{ZnO}$ film has a $(11 \overline{2} 0)$ orientation. The insert of Figure 2 shows the $\phi$-scan from the $\{21 \overline{3} 0\}$ family of reflections for $\mathrm{ZnO}$. We found that the angular separation is $180^{\circ}$, the same as predicted by theory for a single crystal. Also shown is the $\phi$-scan from the $\{12 \overline{3} 5\}$ family of planes from sapphire. The position of the $\{21 \overline{3} 0\}$ peaks from $\mathrm{ZnO}$ and the $\{12 \overline{3} 5\}$ peaks from $\mathrm{Al}_{2} \mathrm{O}_{3}$ coincide in the $\phi$-scans. From this we found that the epitaxial

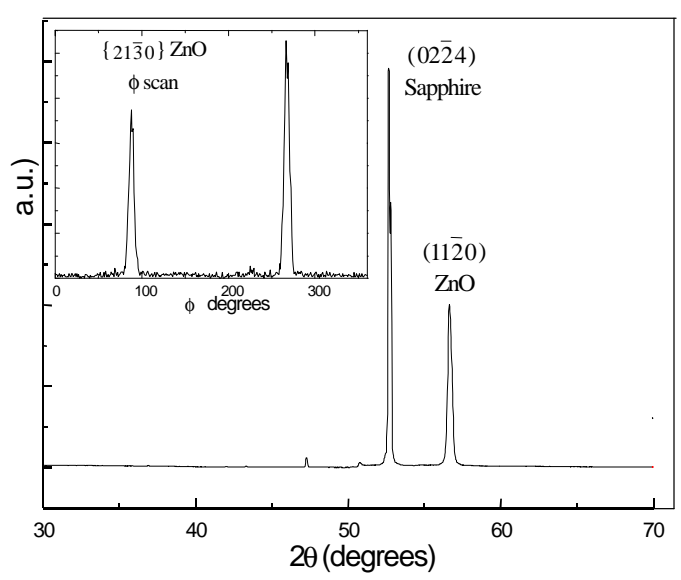

Figure 1: X-ray $2 \theta$ scan, insert: $\phi$-scans of the $\{21 \overline{3} 0\}$ family of planes. relationship for $\mathrm{ZnO}$ films grown on R-plane sapphire is:

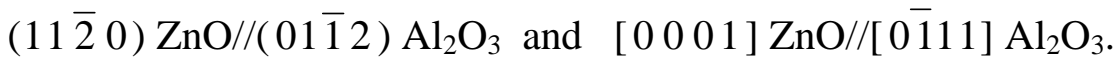

Figure 2 shows a cross-sectional TEM lattice image of the interface between $\mathrm{ZnO}$ and R-sapphire. The interface is observed to be atomically sharp and semicoherent. The $18.3 \%$ misfit along the [11 00 ] direction of $\mathrm{ZnO}$ is relieved by extra half planes on the sapphire side of the interface. On average, there is one dislocation for every five (1 $\overline{1} 00)$ planes. The misfit between the two lattices is completely accommodated by misfit dislocations.

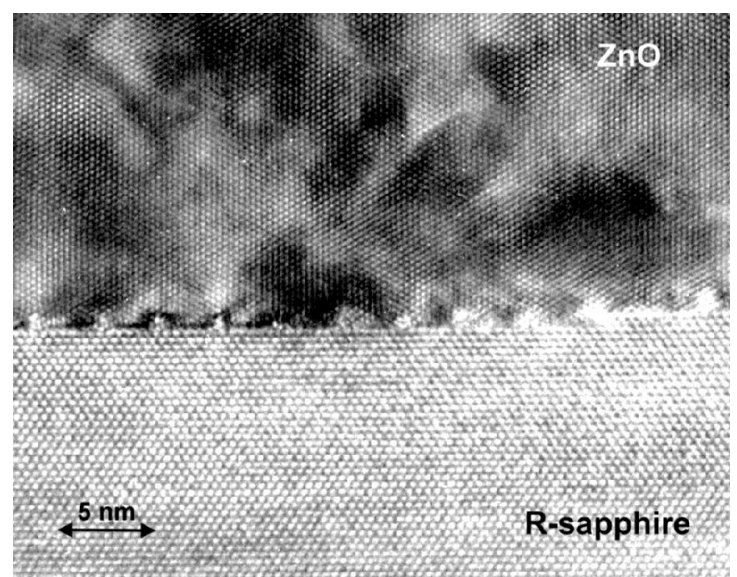

Figure 2: TEM image of the interface between $\mathrm{ZnO}$ and $\mathrm{R}$-sapphire. The view is along the caxis of $\mathrm{ZnO}$. 


\section{OPTICAL PROPERTIES}

Figure 3(a) shows the room temperature PL spectrum. The feature at $3.28 \mathrm{eV}$ is due to bandedge recombination. The broad feature at $2.35 \mathrm{eV}$ (green emission) is the recombination of free electrons with holes via interstitial zinc, or via defects at the grain boundaries[10,11]. From Fig. 3 (a), the ratio of the integrated intensities of the band-edge emission to the deep level emission (broad band green emission) at room temperature is approximately 3:1. Similar results were observed form $\mathrm{ZnO}$ grown by molecular beam epitaxy (MBE)[1].

Figure 3(b) shows the low-temperature PL spectrum collected at $11 \mathrm{~K}$. The peak at $3.363 \mathrm{eV}$ is from donor-bound exciton $\left(\mathrm{D}^{\circ} \mathrm{X}\right)$ transition, while the feature at $3.320 \mathrm{eV}$ is from the acceptor-bound exciton transition. Note that the position of the $\mathrm{D}^{\circ} \mathrm{X}$ is in good agreement with $4 \mathrm{~K}$ PL results from bulk $\mathrm{ZnO}[2]$ indicating that the thin film is almost
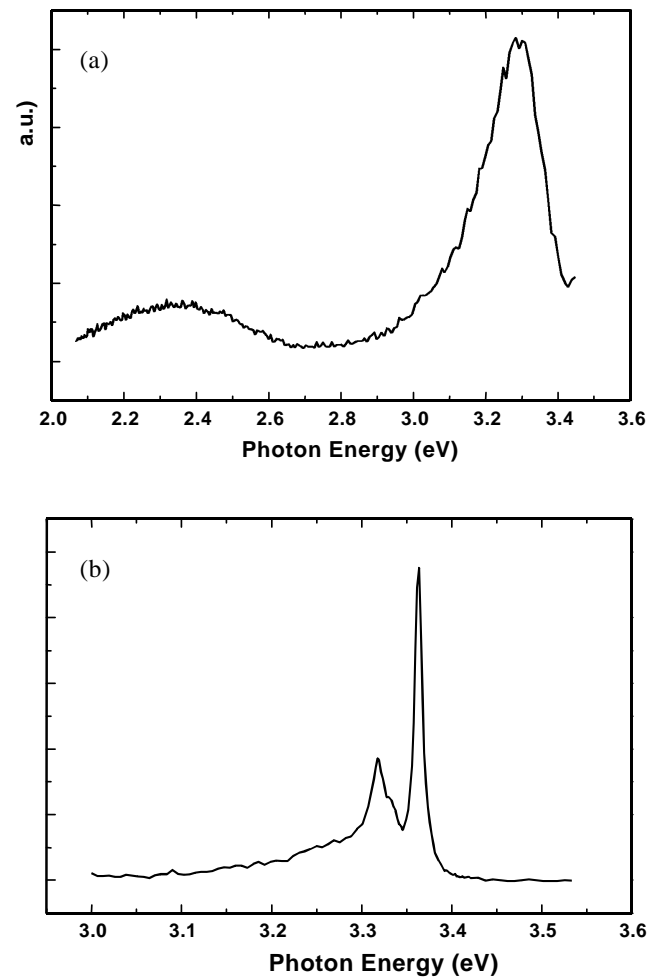

Figure 3: Photoluminescence from $\mathrm{ZnO} / \mathrm{R}-$ sapphire; (a) room temperature, (b) 10k. strain free. The full width at half maximum of this feature is about $6 \mathrm{meV}$, compared to $3 \mathrm{meV}(4.2 \mathrm{~K})$ from bulk $\mathrm{ZnO}$ and $8.9 \mathrm{meV}$ (4.2K) from

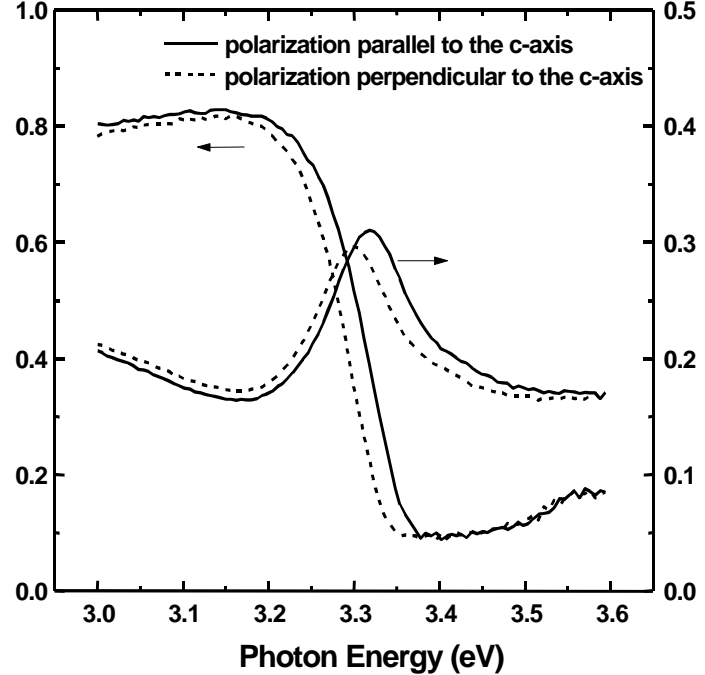

Figure 4: Transmission and Reflectivity of $\mathrm{ZnO}$ on R-plane sapphire as a function of photon energy for light polarized parallel (solid lines) and perpendicular (dashed lines) to the c-axis of the $\mathrm{ZnO}$.
$\mathrm{ZnO}$ grown on $\mathrm{GaN} / \mathrm{SiC}$ by $\mathrm{MBE}$, indicating a film of high quality.

We have observed strong optical anisotropy from the $\mathrm{ZnO}$ film. Figure 4 shows the transmission $T$ and reflectivity $R$ of the $\mathrm{ZnO}$ film measured as a function of photon energy for light polarized parallel and perpendicular to the c-axis of $\mathrm{ZnO}$. Although the shape of the transmission and reflectivity curves are similar for the two polarizations, the curves for $\mathrm{p} / / \mathrm{c}$ are shifted to higher energy by $\sim 20 \mathrm{meV}$ with respect to those for $p \perp c$. This phenomenon is primarily related to the anisotropy in absorption associated with the polarization selection rules[12], combined with the separation in energy of the $\mathrm{C}$ band from $\mathrm{A}$ and $\mathrm{B}$ bands. The energy separation of $\mathrm{A}$ and $\mathrm{B}$ bands is 


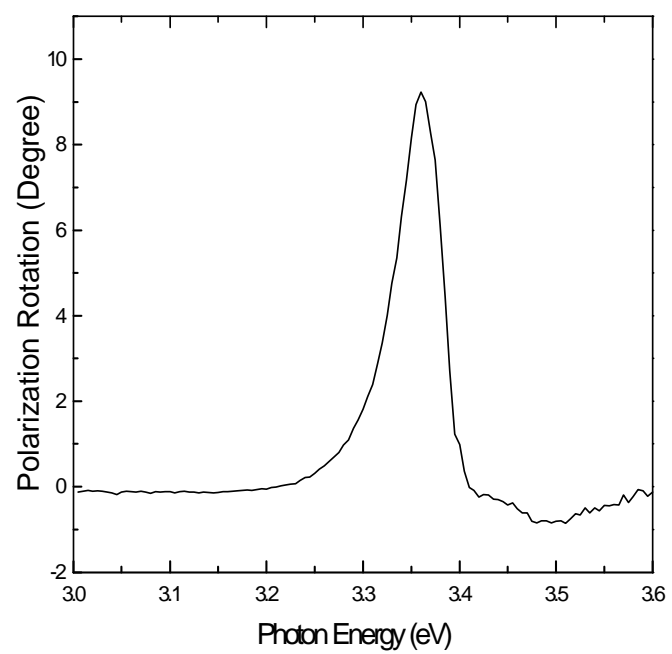

Figure 5: Polarization rotation as a function of photon energy for normal incident light linearly polarized at $45^{\circ}$ with respect to the c-axis. unresolved at room temperature. From a linear fit to the square root singularity, we found that the separation between the $\mathrm{A}(\mathrm{B})$ and $\mathrm{C}$ bands is about $21 \mathrm{meV}$, which is significantly smaller than the $40 \mathrm{meV}$ separation measure in bulk $\mathrm{ZnO}$ at low temperature $(1.6 \mathrm{~K}$ to $4 \mathrm{~K})$. The reason for this is still unclear.

For normal incident light linearly polarized at $45^{\circ}$ with respect to the c-axis, we have observed a large polarization rotation toward the c-axis (Figure 5). This rotation is related to the anisotropic absorption with[13]

$$
\theta=\operatorname{Tan}^{-1}\left[\left(\mathrm{~T}_{/ /} / \mathrm{T}_{\perp}\right)^{1 / 2}\right]-45^{\circ}
$$

where $T_{/ /}$and $T_{\perp}$ are the transmission for $p / / c$ and $p \perp c$, respectively. A maximum rotation of $10^{\circ}$ occurs at $3.335 \mathrm{eV}$. This rotation was used

to demonstrate a high-contrast, high-speed ultraviolet light modulator.

\section{OPTICAL MODULATOR}

We use ultraviolet pulses with a center wavelength corresponding to the static rotation maximum to test the modulator. The polarization of the prove pulse was oriented at $45^{\circ}$ with respect to the c-axis of the $\mathrm{ZnO}$ modulator. Another ultraviolet pulse (pump pulse) is used to control the intensity of the transmitted probe pulse. The transmission of the probe at the photon energy corresponding to the maximum static rotation was monitored as a function of the delay time between the pump and probe pulses. A phase compensator and polarizer placed after the modulator were

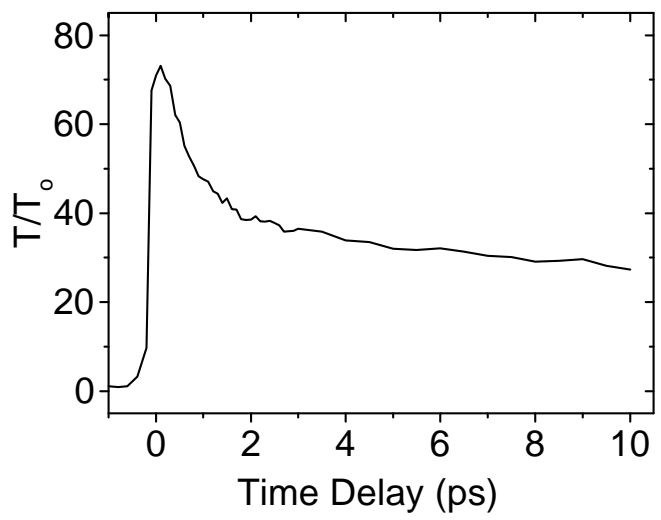

Figure 6: Normalized probe transmission as a function of time delay between the pump and probe pulses.

oriented for minimum transmission of the probe in the absence of the pump[14].

Figure 6 shows the normalized probe transmission $T / T_{o}$ through the crossed polarizer as a function of time delay. $T_{o}$ is the transmission in the absence of the pump. At negative time delays, for which the probe precedes the pump, $T / T_{o}=1$, as expected. As the pump and probe pulses become temporally coincident in the modulator, a sharp, pulse-width limited rise in probe transmission is observed at time delay $\tau=0^{+}$which reaches a maximum more than 70 times 
greater than its value for negative time delays. This peak value in the normalized transmission is defined as the contrast ratio for the device. The pump-induced transmission possesses an initial decay time of $0.75 \mathrm{ps}$, followed by a slower decay to a quasi-equilibrium value within $100 \mathrm{ps}$ (not shown). Subsequent return to equilibrium occurs on a nanosecond time scale. The full width at half maximum of the modulation curve is less than 3 ps.

\section{CONCLUSION}

We have studied the structure and optical properties of high quality epitaxial (11 $\overline{2} 0) \mathrm{ZnO}$ films on $(01 \overline{1} 2)$ sapphire substrates. The epitaxial relationship has been determined. TEM results indicate that the interface is semi-coherent. A sharp, excitonic, band-edge PL peak obtained at $11 \mathrm{~K}$ indicates a strain free film that exhibits excellent quality. We have also observed a strong optical anisotropy that is utilized in a normal incidence, high-contrast, ultraviolet light modulator.

\section{REFEREENCES}

1. M. A. L. Johnson, S. Fujita, W. H. Rowland, Jr., W. C. Huges, J. W. Cook, Jr., and J. F. Schetzina, Journal of Electronic Materials, 25, 855 (1996).

2. F. Hamdani, A. Botchkarev, W. Kim, H. Moorkoc, M. Yeadon, J. M. Gibson, S.-C. Y. Tsen, D. J. Smith, D. C. Reynolds, D. C. Look, K. Evans, C. W. Mitchel and P. Hemenger, Appl. Phys. Lett., 70, 467 (1997).

3. F. Hamdani, M. Yeadon, D. J. Smith, H. Tang, W. Kim, A. Salvador, A. E. Botchkarev, J. M. Gibsor, A. Y. Polyakov, M. Skowronski, and H. Morkoc, J. Appl. Phys. 83, 983 (1998).

4. P. Zu, Z. K. Tang, G. K. L. Wong, M. Kawasaki, A. Ohtomo, H. Koinuma, and Y. Segawa, Solid State Commun., 103, 459 (1997).

5. D. M. Bagnall, Y. F. Chen, Z. Zhu, T. Yao, S. Koyama, M. Y. Shen, and T. Goto, Appl. Phys. Lett, 70, 2230 (1997).

6. V. Srikant, V. Sergo, and D.R. Clarke, J. Am. Cer. Soc. 78, 1931 (1995).

7. P. Roth, and D. F. Williams, J. Appl. Phys. 52, 6685 (1981)

8. B. Cockayne, and P. J. Wright, J. Crystal Growth, 68, 223 (1984).

9. C. R. Gorla, N. W. Emanetoglu, S. Liang, W. E. Mayo, Y. Lu, M. Wraback, and H. Shen, to be published in J. Applied Physics

10. S. Takata, T. Minami and H. Nanto, Jpn. J. Appl. Phys. 20, 1759 (1981).

11. S. Tanaka, K. Takahashi, T. Sekiguchi, K. Sumino, and J. Tanaka, J. Appl. Phys. 77, 4021 (1995).

12. W.Y. Liang and A.D. Yoffe, Physical Review Letters, 20, 59 (1968).

13. H. Shen, M. Wraback, J. Pamulapati, M. Dutta, P.G. Newman, A. Ballato, and Y. Lu, Appl. Phys. Letts. 62, 2908 (1993).

14. M. Wraback, H. Shen, S. Liang, C.R. Gorla, and Y. Lu, to be published in Appl. Phys. Letts. 